
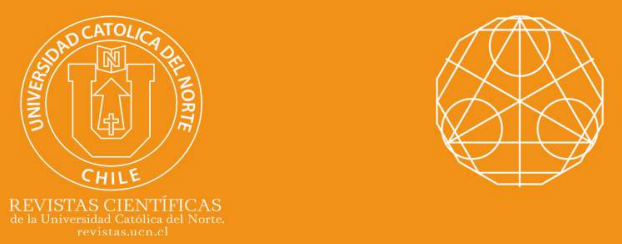

\title{
A generalization of Fibonacci sequence
}

\section{Neeraj Kumar Paul ${ }^{1}$ (1) orcid.org/0000-0003-3963-461X}

Helen K. Saikia² @ orcid.org/0000-0003-1971-9472

Gauhati University, Dept. of Mathematics, Guwahati, AS, India.

1⿴neeraj_dmj@yahoo.co.in ; ${ }^{2}$ hsaikia@yahoo.com

\section{Received: September 2019 | Accepted: May 2020}

\section{Abstract:}

We attempt to generalize Fibonacci sequence by generating certain number of sequences whose terms are obtained by adding the last two generated terms of the preceding sequence. When we consider the particular case of generating only one sequence we obtain the Fibonacci sequence. Moreover we prove some of the results which can be seen as generalized form of the results which hold for Fibonacci sequence. We also verify the results for the particular case of Fibonacci sequence.

Keywords: Fibonacci sequence.

MSC (2020): 11B39.

\section{Cite this article as (IEEE citation style):}

N. K. Paul and H. K. Saikia, "A generalization of Fibonacci sequence", Proyecciones (Antofagasta, On line), vol. 39, no. 6, pp. 1393 1405, Dec. 2020, doi: 10.22199/issn.0717-6279-2020-06-0085.

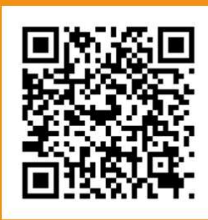

Article copyright: (C) 2020 Neeraj Kumar Paul and Helen K. Saikia. This is an open access article distributed under the terms of the Creative Commons License, which permits unrestricted use and distribution provided the original author and source are credited. 


\section{Introduction}

Fibonacci sequence $\left(F_{k}\right)$ is generated $[1,2]$ by the recursive formula $F_{k}=$ $F_{k-1}+F_{k-2}$ for $k \geq 3$ with $F_{1}=1, F_{2}=1$. That is, each term in the sequence (third term onwards) is the sum of the two that immediately precede it. The Fibonacci sequence is the first known recursive sequence in mathe-

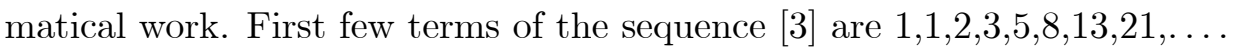

The sequence has been generalized [4] by defining $F_{1}=F_{2}=F_{3}=\ldots=$ $F_{n}=1$ and thereafter each term is the sum of the $n$ terms that immediately precede it. This sequence is called the $n$-nacci sequence and $n=2$ gives the Fibonacci sequence. The limit of the ratio of successive terms of an $n$-nacci series tends to a root of the equation $x+x^{-n}=2$. The limit of the ratio for any $n>0$ is the positive root of the equation $x^{n}-\sum_{i=0}^{n-1} x^{i}=0$.

Another generalization is $(p, q)$ Fibonacci sequence, which is defined as $U(0)=0, U(1)=1, U(k)=p U(k-1)-q U(k-2)$. When $q=-1$, this sequence is called $p$-Fibonacci sequence, for e.g., Pell sequence is also called 2-Fibonacci sequence because $p=2$. The normal Fibonacci sequence is the special case of $p=1$ and $q=-1$.

The above generalizations available in the literature give us a single sequence. In this paper, we generate $m$ number of sequences following certain recursive rules. When the number of sequence is one, i.e. $m=1$, these rules coincide with those generating Fibonacci numbers and we get the Fibonacci Sequence.

We consider $m$ interconnected sequences

$$
\left(S_{1, k}\right),\left(S_{2, k}\right),\left(S_{3, k}\right), \ldots,\left(S_{m, k}\right)
$$

which can be generated according to the following rule

$$
\begin{array}{cl}
S_{1,1}=S_{2,1}=S_{3,1}=\ldots=S_{m, 1}=1, & S_{1,2}=1, \\
S_{i, k}=S_{i-1, k-1}+S_{i-1, k}, & 1<i \leq m, k \geq 2 \\
S_{1, k}=S_{m, k-1}+S_{m, k-2}, & k \geq 3
\end{array}
$$

For $m=1$, we identify the only sequence $\left(S_{1, k}\right)$ as the Fibonacci sequence $\left(F_{k}\right)$.

We generalize the following results along with others which hold for Fibonacci sequence and also verify that they are true for the particular case of Fibonacci sequence when $m=1$. 
- $F_{k}+F_{k+1}=F_{k+2}$

- $\sum_{k=1}^{n} F_{k}=F_{n+2}-1$

- $\sum_{k=0}^{n-1} F_{2 k+1}=F_{2 n}$

- $\sum_{k=1}^{n} F_{2 k}=F_{2 n+1}-1$

Notation: $S_{p, q}$ denotes the $q^{\text {th }}$ term of the $p^{\text {th }}$ sequence. $g c d_{i}\left(S_{i,(p+1) k}\right)$ denotes the greatest common divisor of (multiple of $(p+1))^{\text {th }}$ terms of all the $m$ sequences, i.e, $i$ runs over all the sequences or $i=1,2,3, \ldots, m$.

For e.g., $\operatorname{gcd}_{i}\left(S_{i,(3+1) 2}\right)=\operatorname{gcd}\left(S_{1,8}, S_{2,8}, S_{3,8}, \ldots, S_{m, 8}\right)$.

\section{Generalization of few identities}

Theorem 2.1. Sum of $k^{\text {th }}$ terms in all the sequences and the following term, i.e. $(k+1)^{t h}$ term in the first sequence equals the next i.e. $(k+2)^{t h}$ term in the first sequence.

Symbolically,

$$
\sum_{i=1}^{m} S_{i, k}+S_{1, k+1}=S_{1, k+2}
$$

Fibonacci equivalent: $F_{k}+F_{k+1}=F_{k+2}$

\section{Proof.}

$$
\begin{aligned}
\sum_{i=1}^{m} S_{i, k}+S_{1, k+1} & =\left(S_{1, k}+S_{2, k}+S_{3, k}+\ldots+S_{m-1, k}+S_{m, k}\right)+S_{1, k+1} \\
& =\left(S_{m, k}+S_{m-1, k}+\ldots+S_{3, k}+S_{2, k}+S_{1, k}\right)+S_{1, k+1} \\
& =\left(S_{m, k}+S_{m-1, k}+\ldots+S_{3, k}+S_{2, k}\right)+\left(S_{1, k}+S_{1, k+1}\right) \\
& =\left(S_{m, k}+S_{m-1, k}+\ldots+S_{3, k}+S_{2, k}\right)+S_{2, k+1} \\
& =\left(S_{m, k}+S_{m-1, k}+\ldots+S_{3, k}\right)+\left(S_{2, k}+S_{2, k+1}\right) \\
& =\ldots \\
& =S_{m, k}+S_{m, k+1} \\
& =S_{1, k+2}
\end{aligned}
$$


Columns in the table below show the terms in six sequences (i.e. $m=6$ ). The result is illustrated for $k=2$ and $k=6$, where the summands are bold and the sum is italicized.

\begin{tabular}{|r|r|r|r|r|r|}
\hline 1 & 1 & 1 & 1 & 1 & 1 \\
\hline $\mathbf{1}$ & $\mathbf{2}$ & $\mathbf{3}$ & $\mathbf{4}$ & $\mathbf{5}$ & $\mathbf{6}$ \\
\hline $\mathbf{7}$ & 8 & 10 & 13 & 17 & 22 \\
\hline 28 & 35 & 43 & 53 & 66 & 83 \\
\hline 105 & 133 & 168 & 211 & 264 & 330 \\
\hline $\mathbf{4 1 3}$ & $\mathbf{5 1 8}$ & $\mathbf{6 5 1}$ & $\mathbf{8 1 9}$ & $\mathbf{1 0 3 0}$ & $\mathbf{1 2 9 4}$ \\
\hline $\mathbf{1 6 2 4}$ & 2037 & 2555 & 3206 & 4025 & 5055 \\
\hline 6349 & 7973 & 10010 & 12565 & 15771 & 19796 \\
\hline 24851 & 31200 & 39173 & 49183 & 61748 & 77519 \\
\hline$\ldots$ & $\ldots$ & $\ldots$ & $\ldots$ & $\ldots$ & $\ldots$ \\
\hline
\end{tabular}

When $m=1$, we get,
$\sum_{i=1}^{m} S_{i, k}+S_{1, k+1}=S_{1, k+2}$
or $\quad \sum_{i=1}^{1} S_{i, k}+S_{1, k+1}=S_{1, k+2}$
or
$S_{1, k}+S_{1, k+1}=S_{1, k+2}$
i.e. $F_{k}+F_{k+1}=F_{k+2}$

Theorem 2.2. Sum of first $n$ terms of all the sequences is one less than the $(n+2)^{\text {th }}$ term of the first sequence.

Symbolically, $\sum_{i=1}^{m} \sum_{k=1}^{n} S_{i, k}=S_{1, n+2}-1$

Fibonacci equivalent: $\sum_{k=1}^{n} F_{k}=F_{n+2}-1$

\section{Proof.}

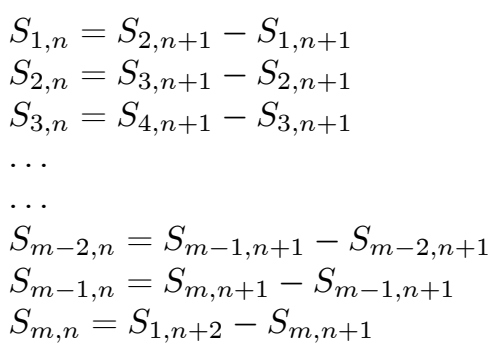

$S_{1, n}=S_{2, n+1}-S_{1, n+1}$

$S_{2, n}=S_{3, n+1}-S_{2, n+1}$

$S_{3, n}=S_{4, n+1}-S_{3, n+1}$

$S_{m-2, n}=S_{m-1, n+1}-S_{m-2, n+1}$

$S_{m, n}=S_{1, n+2}-S_{m, n+1}$ 


\begin{tabular}{|c|c|c|c|c|}
\hline$\cdots$ & $\cdots$ & $\ldots$ & $S_{1,2}=S_{2,3}-S_{1,3}$ & $S_{1,1}=S_{2,2}-S_{1,2}$ \\
\hline & $\ldots$ & $\ldots$ & $S_{2,2}=S_{3,3}-S_{2,3}$ & $S_{2,1}=S_{3,2}-S_{2,2}$ \\
\hline$\cdots$ & $\cdots$ & $\cdots$ & $S_{3,2}=S_{4,3}-S_{3,3}$ & $S_{3,1}=S_{4,2}-S_{3,2}$ \\
\hline$\cdots$ & & & $\ldots$ & $\ldots$ \\
\hline & & & $\stackrel{-}{S}-S \quad S$ & \\
\hline & $\cdots$ & $\cdots$ & $S_{m-2,2}=S_{m-1,3}-S_{m-2,3}$ & $\begin{array}{l}S_{m-2,1}=S_{m-1,2}-S_{m-2,2} \\
S_{m-11}=S_{m}-S_{m-1}\end{array}$ \\
\hline $\begin{array}{l}\cdots \\
\cdots\end{array}$ & $\begin{array}{l}\cdots \\
\cdots\end{array}$ & $\begin{array}{l}\cdots \\
\cdots\end{array}$ & $\begin{array}{l}S_{m-1,2}=S_{m, 3}-S_{m-1,3} \\
S_{m, 2}=S_{1,4}-S_{m, 3}\end{array}$ & $\begin{array}{l}S_{m-1,1}=S_{m, 2}-S_{m-1,2} \\
S_{m, 1}=S_{1,3}-S_{m, 2}\end{array}$ \\
\hline
\end{tabular}

Thus,

$$
\begin{aligned}
& \sum_{i=1}^{m} S_{i, n}=S_{1, n+2}-S_{1, n+1} \quad \sum_{i=1}^{m} S_{i, n-1}=S_{1, n+1}-S_{1, n} \quad \sum_{i=1}^{m} S_{i, n-2}=S_{1, n}-S_{1, n-1} \\
& \ldots \ldots \ldots \sum_{i=1}^{m} S_{i, 2}=S_{1,4}-S_{1,3} \quad \sum_{i=1}^{m} S_{i, 1}=S_{1,3}-S_{1,2}
\end{aligned}
$$

Therefore

$$
\sum_{i=1}^{m} \sum_{k=1}^{n} S_{i, k}=S_{1, n+2}-S_{1,2}=S_{1, n+2}-1
$$

The result is illustrated in the table for $m=6, n=6$, where the summands are bold and the sum is 6348 , which is one less than the italicized term.

\begin{tabular}{|r|r|r|r|r|r|}
\hline $\mathbf{1}$ & $\mathbf{1}$ & $\mathbf{1}$ & $\mathbf{1}$ & $\mathbf{1}$ & $\mathbf{1}$ \\
\hline $\mathbf{1}$ & $\mathbf{2}$ & $\mathbf{3}$ & $\mathbf{4}$ & $\mathbf{5}$ & $\mathbf{6}$ \\
\hline $\mathbf{7}$ & $\mathbf{8}$ & $\mathbf{1 0}$ & $\mathbf{1 3}$ & $\mathbf{1 7}$ & $\mathbf{2 2}$ \\
\hline $\mathbf{2 8}$ & $\mathbf{3 5}$ & $\mathbf{4 3}$ & $\mathbf{5 3}$ & $\mathbf{6 6}$ & $\mathbf{8 3}$ \\
\hline $\mathbf{1 0 5}$ & $\mathbf{1 3 3}$ & $\mathbf{1 6 8}$ & $\mathbf{2 1 1}$ & $\mathbf{2 6 4}$ & $\mathbf{3 3 0}$ \\
\hline $\mathbf{4 1 3}$ & $\mathbf{5 1 8}$ & $\mathbf{6 5 1}$ & $\mathbf{8 1 9}$ & $\mathbf{1 0 3 0}$ & $\mathbf{1 2 9 4}$ \\
\hline 1624 & 2037 & 2555 & 3206 & 4025 & 5055 \\
\hline 6349 & 7973 & 10010 & 12565 & 15771 & 19796 \\
\hline 24851 & 31200 & 39173 & 49183 & 61748 & 77519 \\
\hline$\ldots$ & $\ldots$ & $\ldots$ & $\ldots$ & $\ldots$ & $\ldots$ \\
\hline
\end{tabular}

When $m=1$, we get,

$$
\begin{aligned}
& \sum_{i=1}^{m} \sum_{k=1}^{n} S_{i, k}=S_{1, n+2}-1 \quad \text { or } \quad \sum_{i=1}^{1} \sum_{k=1}^{n} S_{i, k}=S_{1, n+2}-1 \quad \text { or } \\
& \sum_{k=1}^{n} S_{1, k}=S_{1, n+2}-1 \\
& \text { i.e. } \sum_{k=1}^{n} F_{k}=F_{n+2}-1
\end{aligned}
$$


Theorem 2.3. This result is a generalization of Theorem 2.2 above.

$$
\sum_{i=1}^{m} \sum_{k=2}^{n-1} S_{i, k}+\sum_{i=r}^{m} S_{i, 1}+\sum_{i=1}^{r-1} S_{i, n}=S_{r, n+1}-r
$$

Theorem 2.2 is a particular case for $r=1$.

Proof.

$$
\begin{array}{rlrl} 
& & & =S_{1, n+2}-1 \\
& \sum_{i=1}^{m} \sum_{k=1}^{n} S_{i, k} & & =S_{1, n+2}-1 \\
\Rightarrow \quad & \sum_{i=1}^{m} S_{i, 1}+\sum_{i=1}^{m} \sum_{k=2}^{n-1} S_{i, k}+\sum_{i=1}^{m} S_{i, n} & & =S_{1, n+2}-1 \\
\Rightarrow \quad & \left.\quad \sum_{i=1}^{r-1} S_{i, 1}+\sum_{i=r}^{m} S_{i, 1}\right)+\sum_{i=1}^{m} \sum_{k=2}^{n-1} S_{i, k}+\left(\sum_{i=1}^{r-1} S_{i, n}+\sum_{i=r}^{m} S_{i, n}\right) & =S_{1, n+2}-1 \\
\Rightarrow & \sum_{i=1}^{r-1} 1+\sum_{i=r}^{m} S_{i, 1}+\sum_{i=1}^{m} \sum_{k=2}^{n-1} S_{i, k}+\sum_{i=1}^{r-1} S_{i, n}+\sum_{i=r}^{m} S_{i, n} & & =S_{1, n+2}-1-\sum_{i=1}^{r-1} 1-\sum_{i=r}^{m} S_{i, n} \\
\Rightarrow & \sum_{i=r}^{m} S_{i, 1}+\sum_{i=1}^{m} \sum_{k=2}^{n-1} S_{i, k}+\sum_{i=1}^{r-1} S_{i, n} & & =S_{1, n+2}-1-(r-1)-\sum_{i=r}^{m} S_{i, n} \\
\Rightarrow & \sum_{i=r}^{m} S_{i, 1}+\sum_{i=1}^{m} \sum_{k=2}^{n-1} S_{i, k}+\sum_{i=1}^{r-1} S_{i, n} & & =S_{1, n+2}-\sum_{i=r}^{m} S_{i, n}-r
\end{array}
$$

Hence

$$
\begin{aligned}
\sum_{i=1}^{m} \sum_{k=2}^{m-1} S_{i, k}+\sum_{i=r}^{m} S_{i, 1}+\sum_{i=1}^{r-1} S_{i, n} & =\left(S_{m, n}+S_{m, n+1}\right)-\left(\sum_{i=r}^{m-1} S_{i, n}+S_{m, n}\right)-r \\
& =S_{m, n+1}-\sum_{i=r}^{m-1} S_{i, n}-r \\
& =\left(S_{m-1, n}+S_{m-1, n+1}\right)-\left(\sum_{i=r}^{m-2} S_{i, n}+S_{m-1, n}\right)-r \\
& =S_{m-1, n+1}-\sum_{i=r}^{m-2} S_{i, n}-r \\
& \ldots \\
& =S_{r, n+1}-r
\end{aligned}
$$


The result is illustrated in the table for $m=6, n=5$ and $r=4$, where the summands are bold and the sum is 815 , which is 4 (i.e. $r$ ) less than the italicized term.

\begin{tabular}{|r|r|r|r|r|r|}
\hline 1 & 1 & 1 & $\mathbf{1}$ & $\mathbf{1}$ & $\mathbf{1}$ \\
\hline $\mathbf{1}$ & $\mathbf{2}$ & $\mathbf{3}$ & $\mathbf{4}$ & $\mathbf{5}$ & $\mathbf{6}$ \\
\hline $\mathbf{7}$ & $\mathbf{8}$ & $\mathbf{1 0}$ & $\mathbf{1 3}$ & $\mathbf{1 7}$ & $\mathbf{2 2}$ \\
\hline $\mathbf{2 8}$ & $\mathbf{3 5}$ & $\mathbf{4 3}$ & $\mathbf{5 3}$ & $\mathbf{6 6}$ & $\mathbf{8 3}$ \\
\hline $\mathbf{1 0 5}$ & $\mathbf{1 3 3}$ & $\mathbf{1 6 8}$ & 211 & 264 & 330 \\
\hline 413 & 518 & 651 & 819 & 1030 & 1294 \\
\hline 1624 & 2037 & 2555 & 3206 & 4025 & 5055 \\
\hline 6349 & 7973 & 10010 & 12565 & 15771 & 19796 \\
\hline 24851 & 31200 & 39173 & 49183 & 61748 & 77519 \\
\hline$\ldots$ & $\ldots$ & $\ldots$ & $\ldots$ & $\ldots$ & $\ldots$ \\
\hline
\end{tabular}

Theorem 2.4. Sum of all the terms in odd positions (upto $(2 n+1)^{\text {th }}$ position) in all the sequences equals the $(2 n)^{\text {th }}$ term of the last sequence.

Symbolically, $\sum_{i=1}^{m} \sum_{k=0}^{n-1} S_{i, 2 k+1}=S_{m, 2 n}$

Fibonacci equivalent: $\sum_{k=0}^{n-1} F_{2 k+1}=F_{2 n}$

\section{Proof.}

$$
\begin{array}{lcc}
S_{1,1}=S_{2,2}-S_{1,2} & S_{1,3}=S_{2,4}-S_{1,4} & S_{1,5}=S_{2,6}-S_{1,6} \\
\ldots \quad S_{1,2 n-1}=S_{2,2 n}-S_{1,2 n} & & \\
S_{2,1}=S_{3,2}-S_{2,2} & S_{2,3}=S_{3,4}-S_{2,4} & S_{2,5}=S_{3,6}-S_{2,6} \\
\ldots \quad S_{2,2 n-1}=S_{3,2 n}-S_{2,2 n} & & \\
S_{3,1}=S_{4,2}-S_{3,2} & S_{3,3}=S_{4,4}-S_{3,4} & S_{3,5}=S_{4,6}-S_{3,6} \\
\ldots \quad S_{3,2 n-1}=S_{4,2 n}-S_{3,2 n} & \\
\ldots & \ldots & \\
\ldots & \ldots & \\
S_{m-1,1}=S_{m, 2}-S_{m-1,2} & S_{m-1,3}=S_{m, 4}-S_{m-1,4}=S_{m, 6}-S_{m-1,6} \\
\ldots \quad S_{m-1,2 n-1}=S_{m, 2 n}-S_{m-1,2 n} \\
S_{m, 1}=S_{1,3}-S_{m, 2} \quad S_{m, 3}=S_{1,5}-S_{m, 4} & \\
\ldots \quad S_{m, 2 n-1}=S_{1,2 n+1}-S_{m, 2 n} &
\end{array}
$$


Thus,

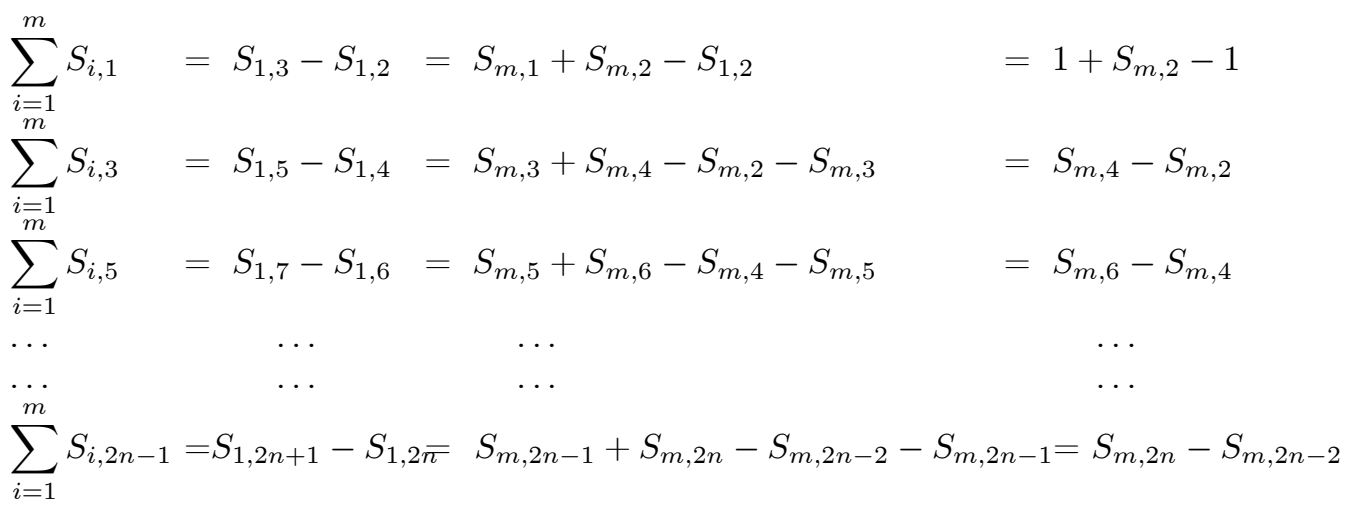

Therefore

$$
\sum_{i=1}^{m} \sum_{k=0}^{n-1} S_{i, 2 k+1}=S_{m, 2 n}
$$

The result is illustrated in the table for $m=6, n=4$, where the summands are bold and the sum is the italicized term.

\begin{tabular}{|r|r|r|r|r|r|}
\hline $\mathbf{1}$ & $\mathbf{1}$ & $\mathbf{1}$ & $\mathbf{1}$ & $\mathbf{1}$ & $\mathbf{1}$ \\
\hline 1 & 2 & 3 & 4 & 5 & 6 \\
\hline $\mathbf{7}$ & $\mathbf{8}$ & $\mathbf{1 0}$ & $\mathbf{1 3}$ & $\mathbf{1 7}$ & $\mathbf{2 2}$ \\
\hline 28 & 35 & 43 & 53 & 66 & 83 \\
\hline $\mathbf{1 0 5}$ & $\mathbf{1 3 3}$ & $\mathbf{1 6 8}$ & $\mathbf{2 1 1}$ & $\mathbf{2 6 4}$ & $\mathbf{3 3 0}$ \\
\hline 413 & 518 & 651 & 819 & 1030 & 1294 \\
\hline $\mathbf{1 6 2 4}$ & $\mathbf{2 0 3 7}$ & $\mathbf{2 5 5 5}$ & $\mathbf{3 2 0 6}$ & $\mathbf{4 0 2 5}$ & $\mathbf{5 0 5 5}$ \\
\hline 6349 & 7973 & 10010 & 12565 & 15771 & 19796 \\
\hline 24851 & 31200 & 39173 & 49183 & 61748 & 77519 \\
\hline$\ldots$ & $\ldots$ & $\ldots$ & $\ldots$ & $\ldots$ & $\ldots$ \\
\hline
\end{tabular}

When $m=1$, we get,

$$
\begin{aligned}
& \sum_{i=1}^{m} \sum_{k=0}^{n-1} S_{i, 2 k+1}=S_{m, 2 n} \quad \text { or } \quad \sum_{i=1}^{1} \sum_{k=0}^{n-1} S_{i, 2 k+1}=S_{1,2 n} \quad \text { or } \\
& \sum_{k=0}^{n-1} S_{1,2 k+1}=S_{1,2 n} \text { i.e. } \sum_{k=0}^{n-1} F_{2 k+1}=F_{2 n}
\end{aligned}
$$

Corollary 2.4.1. Sum of all the terms in even positions (upto $(2 n)^{\text {th }}$ position) in all the sequences is one less than the $(2 n+1)^{\text {th }}$ term of the last 
sequence.

Symbolically, $\sum_{i=1}^{m} \sum_{k=1}^{n} S_{i, 2 k}=S_{m, 2 n+1}-1$

Fibonacci equivalent: $\sum_{k=1}^{n} F_{2 k}=F_{2 n+1}-1$

Proof. $\quad \sum_{i=1}^{m} \sum_{k=1}^{n} S_{i, 2 k}=\sum_{i=1}^{m} \sum_{k=1}^{2 n} S_{i, k}-\sum_{i=1}^{m} \sum_{k=0}^{n-1} S_{i, 2 k+1}=S_{1,2 n+2}-1-$ $S_{m, 2 n}=S_{m, 2 n+1}-1$

The result is illustrated in the table for $m=6, n=3$, where the summands are bold and the sum is 5054, which is one less than the italicized term.

\begin{tabular}{|r|r|r|r|r|r|}
\hline 1 & 1 & 1 & 1 & 1 & 1 \\
\hline $\mathbf{1}$ & $\mathbf{2}$ & $\mathbf{3}$ & $\mathbf{4}$ & $\mathbf{5}$ & $\mathbf{6}$ \\
\hline 7 & 8 & 10 & 13 & 17 & 22 \\
\hline $\mathbf{2 8}$ & $\mathbf{3 5}$ & $\mathbf{4 3}$ & $\mathbf{5 3}$ & $\mathbf{6 6}$ & $\mathbf{8 3}$ \\
\hline 105 & 133 & 168 & 211 & 264 & 330 \\
\hline $\mathbf{4 1 3}$ & $\mathbf{5 1 8}$ & $\mathbf{6 5 1}$ & $\mathbf{8 1 9}$ & $\mathbf{1 0 3 0}$ & $\mathbf{1 2 9 4}$ \\
\hline 1624 & 2037 & 2555 & 3206 & 4025 & 5055 \\
\hline 6349 & 7973 & 10010 & 12565 & 15771 & 19796 \\
\hline 24851 & 31200 & 39173 & 49183 & 61748 & 77519 \\
\hline$\ldots$ & $\ldots$ & $\ldots$ & $\ldots$ & $\ldots$ & $\ldots$ \\
\hline
\end{tabular}

When $m=1$, we get,

$\sum_{i=1}^{m} \sum_{k=1}^{n} S_{i, 2 k}=S_{m, 2 n+1}-1 \quad$ or $\quad \sum_{i=1}^{1} \sum_{k=1}^{n} S_{i, 2 k}=S_{1,2 n+1}-1 \quad$ or

$\sum_{k=1}^{n} S_{1,2 k}=S_{1,2 n+1}-1$

i.e. $\sum_{k=1}^{n} F_{2 k}=F_{2 n+1}-1$

Theorem 2.5. $\lim _{k \rightarrow \infty} \frac{S_{i, k}}{S_{i, k+1}}$ is a root of the polynomial $x(x+1)^{m}-1$ for $i=1,2, \ldots, m$.

For Fibonacci sequence, $m=1, i=1$ and therefore $\lim _{k \rightarrow \infty} \frac{S_{1, k}}{S_{1, k+1}}=$ $\lim _{k \rightarrow \infty} \frac{F_{k}}{F_{k+1}}=(\text { Golden ratio })^{-1}$, which is a root of the polynomial $x(x+1)-1$. 
Proof. Suppose $\lim _{k \rightarrow \infty} \frac{S_{i, k}}{S_{i, k+1}}=l$.

Then

$$
\lim _{k \rightarrow \infty} \frac{S_{i+1, k}}{S_{i+1, k+1}}=\lim _{k \rightarrow \infty} \frac{S_{i, k-1}+S_{i, k}}{S_{i, k}+S_{i, k+1}}=\lim _{k \rightarrow \infty} \frac{\frac{S_{i, k-1}}{S_{i, k}}+1}{1+\frac{S_{i, k+1}}{S_{i, k}}}=\frac{l+1}{1+\frac{1}{l}}=l
$$

Thus, $\lim _{k \rightarrow \infty} \frac{S_{1, k}}{S_{1, k+1}}=\lim _{k \rightarrow \infty} \frac{S_{2, k}}{S_{2, k+1}}=\ldots=\lim _{k \rightarrow \infty} \frac{S_{m, k}}{S_{m, k+1}}=l$.

Also

$$
\lim _{k \rightarrow \infty} \frac{S_{i, k}}{S_{i+1, k}}=\lim _{k \rightarrow \infty} \frac{S_{i, k}}{S_{i, k-1}+S_{i, k}}=\lim _{k \rightarrow \infty} \frac{1}{\frac{S_{i, k-1}}{S_{i, k}}+\frac{S_{i, k}}{S_{i, k}}}=\frac{1}{l+1}
$$

Now combining the rules for the interconnected sequences, we get,

$$
\begin{aligned}
& \qquad \begin{aligned}
S_{1, k+1} & =S_{m, k-1}+S_{m, k} \\
& =\ldots \\
& =a_{m} S_{1, k}+a_{m-1} S_{1, k-1}+a_{m-2} S_{1, k-2}+\ldots+a_{0} S_{1, k-m}
\end{aligned} \\
& \text { where } a_{i}=\left(\begin{array}{c}
m \\
i
\end{array}\right) .
\end{aligned}
$$

Thus,

$$
\begin{aligned}
& \lim _{k \rightarrow \infty} \frac{S_{1, k-1}}{S_{1, k}}=\lim _{k \rightarrow \infty} \frac{S_{1, k}}{S_{1, k+1}} \\
& \Rightarrow \lim _{k \rightarrow \infty} \frac{S_{1, k-1}}{S_{1, k}}=\lim _{k \rightarrow \infty} \frac{S_{1, k}}{a_{m} S_{1, k}+a_{m-1} S_{1, k-1}+a_{m-2} S_{1, k-2}+\ldots+a_{0} S_{1, k-m}} \\
& \Rightarrow \lim _{k \rightarrow \infty} \frac{S_{1, k-1}}{S_{1, k}}=\lim _{k \rightarrow \infty} \frac{1}{a_{m} \frac{S_{1, k}}{S_{1, k}}+a_{m-1} \frac{S_{1, k-1}}{S_{1, k}}+a_{m-2} \frac{S_{1, k-2}}{S_{1, k}}+\ldots+a_{0} \frac{S_{1, k-m}}{S_{1, k}}}
\end{aligned}
$$

or,

$l=\frac{1}{a_{m}+a_{m-1} l+a_{m-2} l^{2}+\ldots+a_{0} l^{m}} \quad \Rightarrow l=\frac{1}{(1+l)^{m}} \quad \Rightarrow l(1+l)^{m}=1$

Therefore $l$ is a root of the polynomial $x(x+1)^{m}-1$.

Corollary 2.5.1. $\lim _{k \rightarrow \infty} \frac{S_{i, k+1}}{S_{i, k}}$ is a root of the polynomial $x^{m+1}-(x+1)^{m}$ for $i=1,2, \ldots, m$.

For Fibonacci sequence, $m=1, i=1$ and therefore $\lim _{k \rightarrow \infty} \frac{S_{1, k+1}}{S_{1, k}}=\lim _{k \rightarrow \infty} \frac{F_{k}+1}{F_{k}}=$ Golden ratio, which is a root of the polynomial $x^{2}-(x+1)$. 
Proof. The proof is obvious.

Theorem 2.6. If $m=p-1, p$ prime, then $p \mid g c d_{i}\left(S_{i,(p+1) k}\right), k \geq 1$.

Suppose $p=2$. Then $m=1$ i.e. we have the Fibonacci sequence. In this case, $2 \mid \operatorname{gcd}\left(S_{1,3 k}\right)$ i.e. $2 \mid S_{1,3 k}$ i.e. $2 \mid F_{3 k}$. In other words, every third term in Fibonacci sequence is even.

Proof. For prime $p, p \mid\left(\begin{array}{c}p-1 \\ i\end{array}\right)+\left(\begin{array}{c}p-1 \\ i+1\end{array}\right)$ because $\left(\begin{array}{c}p-1 \\ i\end{array}\right)+\left(\begin{array}{c}p-1 \\ i+1\end{array}\right)=\left(\begin{array}{c}p \\ i+1\end{array}\right)=\frac{p !}{(i+1) !(p-i-1) !}$.

Thus $p \mid\left(\begin{array}{c}m \\ i\end{array}\right)+\left(\begin{array}{c}m \\ i+1\end{array}\right)$.

Now,

$$
S_{1, k+1}=\left(\begin{array}{c}
m \\
m
\end{array}\right) S_{1, k}+\left(\begin{array}{c}
m \\
m-1
\end{array}\right) S_{1, k-1}+\left(\begin{array}{c}
m \\
m-2
\end{array}\right) S_{1, k-2}+\ldots+\left(\begin{array}{c}
m \\
0
\end{array}\right) S_{1, k-m}
$$

with $S_{1,1}=1, S_{1, q}=0, q \leq 0$.

Hence, we get

$\begin{array}{lll}S_{1,1}=1(\bmod p), & S_{1,2}=1(\bmod p), & S_{1,3}=\left(\begin{array}{c}m \\ 1\end{array}\right)+\left(\begin{array}{c}m \\ 0\end{array}\right)(\bmod p), \\ S_{1,4}=\left(\begin{array}{c}m \\ 2\end{array}\right)+\left(\begin{array}{c}m \\ 1\end{array}\right)(\bmod p), & \ldots \ldots \ldots \ldots \ldots, & S_{1, m+2}=\left(\begin{array}{c}m \\ m\end{array}\right)+\left(\begin{array}{c}m \\ m-1\end{array}\right)(\bmod p)\end{array}$

That is,

$$
\begin{gathered}
S_{1,1}=1(\bmod p), \quad S_{1,2}=1(\bmod p), \quad S_{1,3}=0(\bmod p), \quad S_{1,4}=0(\bmod p), \\
\ldots, S_{1, m+2}=0(\bmod p)
\end{gathered}
$$

Therefore,

$S_{1, m+3}=1(\bmod p), S_{1, m+4}=1(\bmod p), S_{1, m+5}=0(\bmod p), S_{1, m+6}=$ $0(\bmod p), \ldots, S_{1,2(m+2)}=0(\bmod p)$

By the sequence generating rule, we get,

$S_{1,2}=1(\bmod p)$,

$S_{1,3}=0(\bmod p), \quad S_{2,3}=1(\bmod p)$,

$S_{1,4}=0(\bmod p), \quad S_{2,4}=0(\bmod p), \quad S_{3,4}=1(\bmod p)$, 


$$
\begin{array}{lll}
S_{1,5}=0(\bmod p), & S_{2,5}=0(\bmod p), & S_{3,5}=0(\bmod p), \\
\ldots & \ldots & \ldots \\
\ldots & \ldots & \ldots \\
S_{1, m+2}=0(\bmod p), & S_{2, m+2}=0(\bmod p), & S_{3, m+2}=0(\bmod p), \\
& \ldots & S_{m, m+2}=0(\bmod p)
\end{array}
$$

and so on. Thus $S_{i, m+2}=0(\bmod p) \forall i$.

That is $p \mid S_{i, m+2} \forall i$, or $p \mid S_{i, p+1} \forall i$. Hence $p \mid g c d_{i}\left(S_{i,(p+1)}\right)$.

Finally, using the expression for $S_{1, k+1}$ provided in the beginning of this proof, we can easily prove that $p \mid g c d_{i}\left(S_{i,(p+1) k}\right), k \geq 1$.

Theorem 2.7. If $m=p^{\alpha}-1, p$ prime, then $p \mid \operatorname{gcd}_{i}\left(S_{i,\left(p^{\alpha}+1\right) k}\right), k \geq 1$.

Proof. The proof is in the same line as in case of Theorem 2.6 using the fact that for prime $p, p \mid\left(\begin{array}{c}p^{\alpha}-1 \\ i\end{array}\right)+\left(\begin{array}{c}p^{\alpha}-1 \\ i+1\end{array}\right)$.

\section{Conclusion}

The generalization of Fibonacci sequence as discussed in this paper, has opened a new wider scope to explore all the results involving Fibonacci sequence. Binet's Formula, Cassini's identity etc. can be explored to be generalized accordingly. The last two results dealt with the gcd of the terms in the $m$ sequences generated for the particular cases when $m+1$ is a positive power of a prime $p$. We conclude this paper with an open problem which deals with the general case when $m+1=p_{1}{ }^{\alpha_{1}} p_{2}{ }^{\alpha_{2}} \ldots p_{t}{ }^{\alpha_{t}}$.

Problem: If $m=p_{1}{ }^{\alpha_{1}} p_{2}{ }^{\alpha_{2}} \ldots p_{t}{ }^{\alpha_{t}}-1$, where $p_{i}$ 's are primes and $t \geq 2$, then is it true that $\operatorname{gcd}_{i}\left(S_{i, k}\right)=1, \forall k$ ?

\section{Acknowledgement}

The first author thanks University Grants Commission of India for its financial support to the work. 


\section{References}

[1] D. M. Burton, Elementary number theory, 7th ed. Boston, MA: McGraw-Hill, 2011.

[2] G. H. Hardy and E. M. Wright, An introduction to the theory of numbers, 6th ed. Oxford: Oxford University Press, 2008.

[3] N. J. A. Sloane, "A000045"," The On-Line Encyclopedia of Integer Sequences, 26-Oct-2020. [Online]. Available: https:/ / oeis.org/ A000045

[4] "Generalizations of Fibonacci numbers", Wikipedia, 2020. [On line]. Available: https:/ / en.wikipedia.org/ wiki/ Generalizations_of_Fibonacci_numbers 